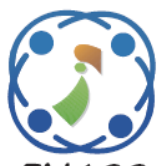

\title{
Efficiency Enhancement of a Fixed Photovoltaic Panel Using Simple Motorized Reflection Model
}

\author{
Hicham Bouzakri $^{1 *} \quad$ Ahmed Abbou $^{1} \quad$ Khalid Chennoufi $^{1}$ \\ ${ }^{1}$ Electrical Engineering Department, Mohammadia School of Engineers, \\ Mohamed V University in Rabat, Morocco, \\ * Corresponding author's Email: hichambouzakri@research.emi.ac.ma
}

\begin{abstract}
The optimal installation of a photovoltaic panel in fixed mode, allows us to receive the maximum of solar radiation at noon, when the sun is in front of the panel, although the output drops in the morning and afternoon. In order to maintain this efficiency as much as possible, the photovoltaic panel must be oriented by a solar tracker. However, the introduction of such a mechanism makes the installation expensive and complicated during maintenance, especially since a part of the energy produced by the panel will itself be consumed for its operation. This paper proposes a simple and less expensive system allowing the photovoltaic panel in fixed mode to maintain its efficiency almost at the maximum all day long with the introduction of a simple reflection system based on two reversible mirrors at noon. After a theoretical study and on the basis of a simulation, the proposed system will have an efficiency of more than about $35 \%$ compared to that in fixed mode and more than $90 \%$ compared to that in tracking mode, these results will be validated via an experiment at the end of the document with a prototype produced.
\end{abstract}

Keywords: Fixed panel, Sun tracker, Photovoltaic panel, Solar tracking, Mirror, Solar radiation.

\section{Introduction}

Energy is said to be "renewable" when its source is natural and inexhaustible. Either because it is able to replenish itself naturally, or because it is available in unlimited quantities on a human scale, like solar energy or wind. Photovoltaic energy is one of the renewable energies widely used in the world in recent decades. It consists of transforming solar radiation into electricity via photovoltaic panels [1,2]. In order to maximize the efficiency of the cells of the latter, they must be oriented perpendicular to direct solar radiation. This is why we add a mechanism called a solar tracker.

A mono-axial [3-5] or bi-axial solar tracker [6-8] is a system that keeps the orientation of the photovoltaic panel towards the sun as much as possible. However, the addition of this device makes the system on the one hand expensive and on the other hand consumable from the energy point of view.

Researchers in Jordan made an electric power efficiency comparison of the different types of solar trackers with a fixed photovoltaic panel mode. The results show an increase of $43.87 \%$ for a two-axis tracker and between $15.69 \%$ and $37.53 \%$ for singleaxis trackers depending on the orientation of the tracking axis [9].

On 2019 we proposed via a theoretical study, an equatorial mono-axial solar tracker model that allows the solar panel to receive between $91.7 \%$ and $100 \%$ (depending on the season) of direct solar irradiation compared to what receives a surface normal to solar radiation. The realization of the experiment showed that the proposed model offers an efficiency of about $49 \%$ compared to a photovoltaic panel in fixed mode [10]. An improvement made to this model in 2020 by the addition of a reflective system, makes the efficiency of our equatorial axial mono tracker very close in production to a two-axis tracker [11]. Nevertheless, the cost of production and the complicity of maintenance and the energy consumption of the entire system remain major drawbacks of solar trackers. A solar panel in fixed mode (orientation to the south with an inclination equal to the latitude of the place) receives the maximum solar radiation at noon, when the sun 
reaches the meridian, although in the morning or in the afternoon the yield falls as the sun moves away from the meridian.

The main idea of this document is to increase the daily output of a photovoltaic panel in fixed mode when the sun moves away from the meridian, either in the morning or in the afternoon. And this by adding a simple reflective system, less expensive and negligible energy consumption.

A study by Japanese researchers has shown that adding a reflective system at the bottom of a solar collector can increase its absorption by $20 \%$ to $33 \%$ depending on the size of the reflector [12]. Researchers in India proposed to add a mirror reflective system with an optimal tilt of $40^{\circ}$ for summer and $15^{\circ}$ for winter, the results showed an improvement in power of 10 to $19.84 \%$ in summer and from 10 to $13.23 \%$ in winter [13], the major drawback of this system is the change of the tilt angle twice a year during winter and summer. Another reflective model proposed by other researchers with the use of two mirrors at the bottom and top without changing the tilt angle, was able to achieve a $15 \%$ increase in power output [14], we notice that the size of the two mirrors and their inclination compared to the photovoltaic panel makes the model voluminous, which will complicate the installation of several models in series. The quarter mirror model was thus proposed by researchers who were able to increase the efficiency of the photovoltaic panel by around $30 \%$ [15]. According to these studies, the researchers introduced to the photovoltaic panel a system of reflections without follow-up, that is to say they kept the whole model in fixed mode in order to increase the production of energy without consumption, this increase remains limited. with the occupation of a large area around the solar panel. A reflective tracking system was developed by researchers in India showing an increase in production of around $30 \%$ [16]. The use of a tracking system for a reflector increases the solar radiation incident on the panel; therefore the system dedicates part of the energy produced for consumption.

This document proposes a new model of the photovoltaic panel in fixed mode based on the reflection; it consists in adding two mirrors in the East and West of the perpendicular solar panel. The movement of the two mirrors is reversible, i.e., if one is activated the other is deactivated and vice versa. Thus, we will avoid that the model will be large and therefore occupy more space compared to the models mentioned above. The size of each mirror is calculated in such a way as to keep the amount of solar irradiation incident on the photovoltaic panel in fixed mode close to the size of a mirror with a perfect tracking system (surface normal to solar radiation). The model has a simple motorization system that allows the mirrors to be reversed at noon. The interest of this study is to develop a simple and mountable model with any fixed photovoltaic panel. The model allows the latter to produce an amount of energy close to that produced by a solar tracker PV with negligible consumption and at a lower cost. We will first present the modeling of the system via a theoretical study allowing calculating the dimension of each mirror. Then we will make a comparison through a theoretical study and a simulation of the amount of direct solar irradiation incident on the PV with and without the proposed model, in order to analyze and evaluate the performance of the entire model. The results found will be compared with the same PV in dual axis tracking mode. And in the last step we will make a real prototype by which we will apply the necessary tests in order to enhance the performance of the proposed model.

\section{The position of the sun in relation to a fixed photovoltaic panel}

We all know that the earth revolves around it in 24 hours and that the axis of rotation of the earth at the northern hemisphere is oriented towards the pole star and in the southern hemisphere towards a place close to the star Sigma Octantis, which is illustrated in Fig. 1.

From the Fig. 2, at the equinoxes (September and March Equinox), the sun rises exactly from geographic east and sets in geographic west. On equinox days (September 22/23 or March 20/21), the surface of a photovoltaic panel in fixed mode (orientation towards the south with an inclination equal to the altitude of the place) receives the maximum direct solar irradiation, since it is oriented towards the celestial equator and the sun on these days also moves on the celestial equator (apparent movement of the sun) [17].

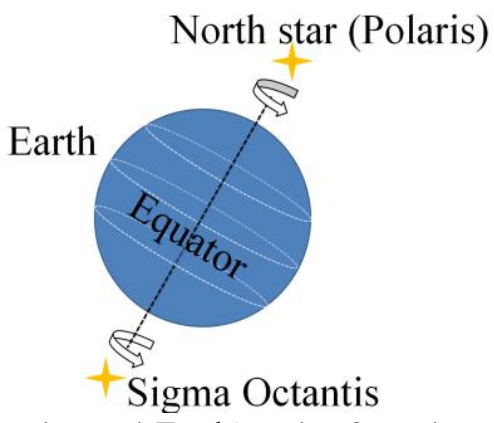

Figure. 1 Earth's axis of rotation 


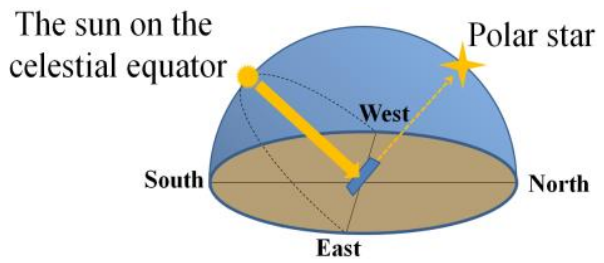

Figure. 2 The orientation of PV in fixed mode towards the celestial equator

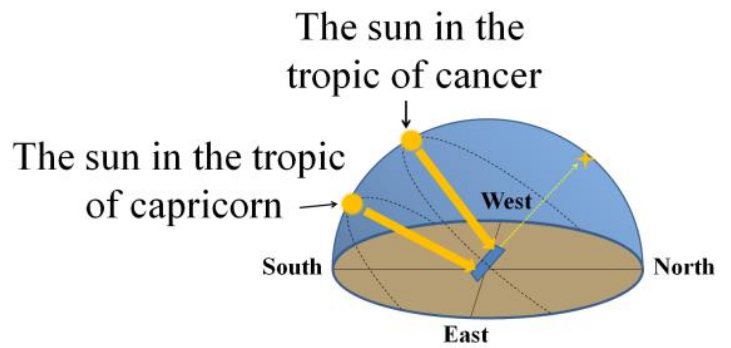

Figure. 3 Direct solar radiation versus fixed PV at winter and summer solstice

From the Fig. 2, at the equinoxes (September and March Equinox), the sun rises exactly from geographic east and sets in geographic west. On equinox days (September 22/23 or March 20/21), the surface of a photovoltaic panel in fixed mode (orientation towards the south with an inclination equal to the altitude of the place) receives the maximum direct solar irradiation, since it is oriented towards the celestial equator and the sun on these days also moves on the celestial equator (apparent movement of the sun) [17].

As the solar path descends to the Tropic of Capricorn or rises to the Tropic of Cancer, the tilt angle of direct solar radiation increases and therefore the amount of solar radiation received by the panel decreases, which is illustrated in Fig. 3 [17].

\section{The proposed model}

Before starting the modeling of the proposed system, we will calculate for an equinox day the percentage of the amount of direct solar irradiation received by a surface in fixed mode (orientation to the south with an inclination equal to the altitude of the place) compared to that received by a surface normal to solar radiation (surface with a perfect solar tracker as shown in Fig. 4) [17].

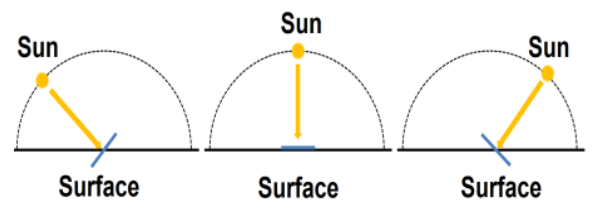

Figure. 4 The surface normal to solar radiation

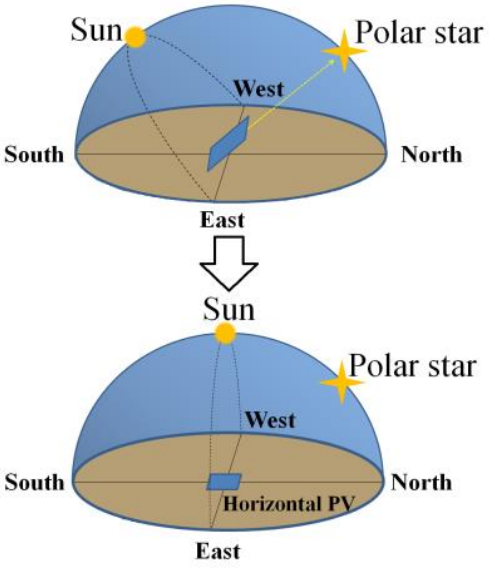

Figure. 5 PV fixed on the day of equinox similar to horizontal PV

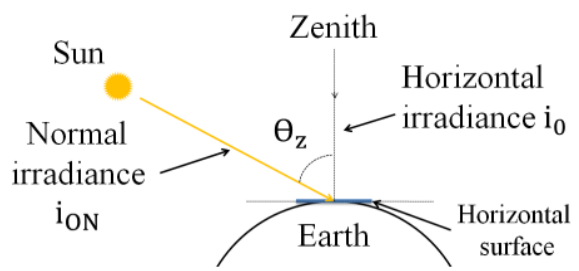

Figure. 6 Relationship between the direct normal and the horizontal irradiance [17]

Since we are interested in direct solar radiation and in order to simplify the theoretical study, we will proceed with the calculations outside the atmosphere. On the day of the equinox, a surface oriented to the south with an inclination equal to the altitude of the place, is considered as a horizontal surface with a solar path which passes through the zenith, which is illustrated in Fig. 5.

From the Fig. 6, the amount of a direct irradiation received by a horizontal surface is calculated by the following relationship [17]:

$$
I_{o}=I_{\text {on }} \cos \theta_{z}
$$

$\theta_{\mathrm{z}}$ is the angle of inclination of the sun in relation to the zenith, in the case of an equinox day this angle varies between $-90^{\circ}$ at sunrise and $+90^{\circ}$ at sunset. According to Eq. (1) on the day of the equinox, the amount of direct solar irradiation from an inclined surface $I_{0}$ relative to that of a surface normal to solar radiation $\mathrm{I}_{\mathrm{on}}$ on is calculated by the following relationship:

$$
I_{o}=I_{o n} \frac{\sum_{\theta_{z}=-90^{\circ}}^{+90^{\circ}}\left(\cos \theta_{z}\right)}{180^{\circ}}=63.5 \% I_{o n}
$$

According to Eq. (1), if $\theta_{\mathrm{z}}= \pm 60^{\circ}$ we will have $\mathrm{I}_{\mathrm{o}}=0.5 \mathrm{I}_{\mathrm{on}}$ i.e. as shown in Fig. 7 when the sun rises 


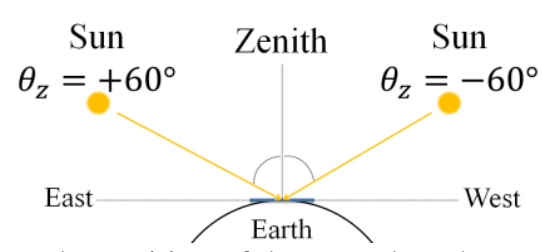

Figure. 7 The position of the sun when the PV surface receives half of solar irradiation relative to the normal radiating surface

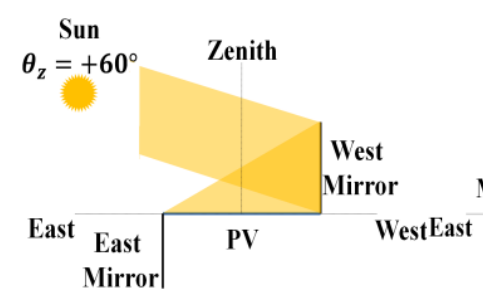

(a)

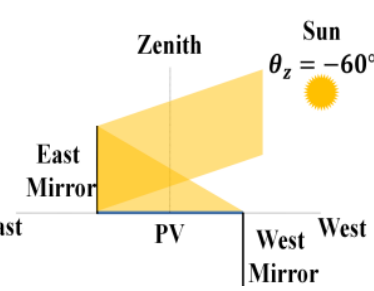

(b)
Figure. 8 (a) The addition of solar radiation through the western mirror and (b) The addition of solar radiation through the eastern mirror

$30^{\circ}$ in the morning, compared to the horizon or goes down to $30^{\circ}$ at bedtime, the amount of solar irradiation that the inclined surface receives on the

equinox day (or a horizontal surface), is equal to half that the same surface can receive in tracking mode (50\% compared to a normal surface to solar radiation).

In order to compensate for this lost amount, the idea is to add two mirrors perpendicular to our tilted surface, one to the west of the panel, the other to the east. The 1 st western mirror is dedicated to the morning when $0^{\circ}<\theta_{\mathrm{z}}<+90^{\circ}$, while the 2nd eastern mirror is dedicated to the afternoon when $0^{\circ}<\theta_{\mathrm{z}}<-90^{\circ}$.. The position of the two mirrors will be invertible at noon, that is to say that when we are in the morning the 1st mirror is present at the top while the 2nd is absent at the bottom as shown in Fig. 8 (a), and the on the contrary, the first descends under the panel in the afternoon, leaving the role to the second who goes up as shown in Fig. 8 (b). In this case the 1st mirror compensates for what is lost in the morning and the 2nd for what is lost in the afternoon. The movement of these two mirrors will be controlled by a small motor.

\subsection{The size of each mirror}

According to Fig. 9, the length of each mirror (E) is a width of PV, so we will calculate the width of the mirrors (C) compared to the length of the PV panel (A), in order to compensate for the $50 \%$ radiation lost at $\theta_{\mathrm{z}}= \pm 60^{\circ}$.

We will consider that the mirrors have a rate of reflections $\mathrm{R}=1$ (total reflections of radiation).

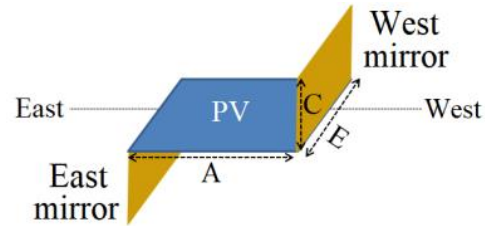

Figure. 9 The width and length of each mirror in relation to the $\mathrm{PV}$

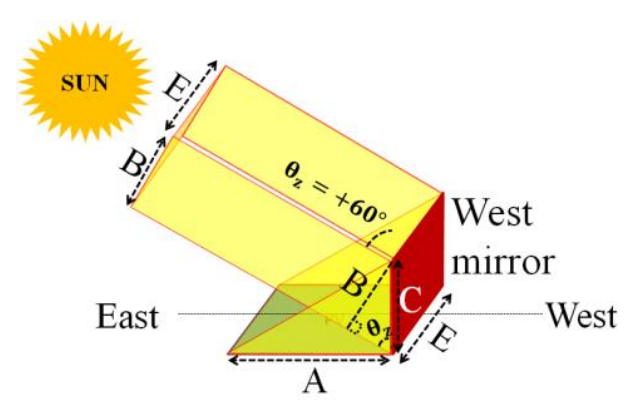

Figure. 10 The path of solar radiation to $\theta_{z}=+60^{\circ}$

Take the case of the morning; the mirror that will be functional is the one to the west of the panel, as shown in Fig. 10.

According to Eq. (1) if $\theta_{z}=60^{\circ}$, we will have $I_{o}=0.5 I_{o n}$. In order to compensate for the $50 \%$ lost, the amount of solar radiation reflected by the western mirror on the panel (shown in Fig. 10 by the area BE) must equal half of the area of PV (shown in Fig. 10 by the surface $\frac{\mathrm{AE}}{2}$ ), then: $\mathrm{BE}=0.5 \mathrm{AE}$ therefore $\mathrm{B}=$ $0.5 \mathrm{~A}$

According to the same Fig. 10, we have:

$$
\sin \theta_{z}=\sin 60^{\circ}=\frac{B}{C}=\frac{0.5 A}{C}
$$

So:

$$
C=0.577 A
$$

\subsection{The amount of solar irradiation added by the western mirror compared to $I_{o n}$}

3.2.1. When $+60^{\circ}<\theta_{z}<+90^{\circ}$

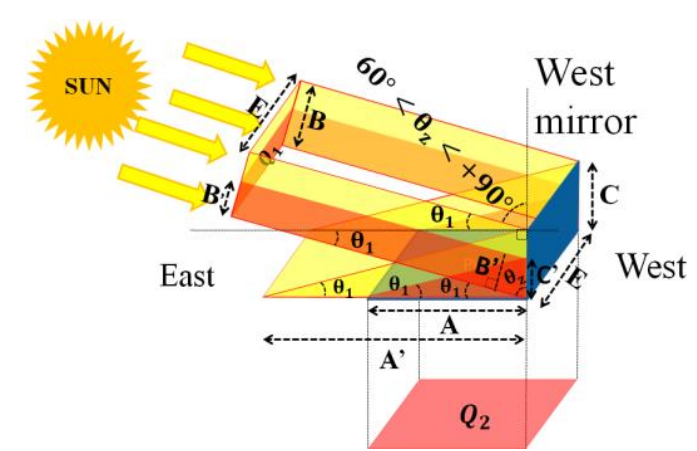

Figure. 11 The solar radiation if $60^{\circ}<\theta_{z}<+90^{\circ}$ 
At $+60^{\circ}<\theta_{z}<+90^{\circ}$, the direct solar radiation reflected by the mirror (represented in figure 11 by the surface $\mathrm{BE}$ ) covers an area more than the surface of the panel (represented in Fig. 11 by $A^{\prime} E$ ). The panel receives just the amount of direct solar radiation that comes from the surface $Q_{1}=B^{\prime} E$. So the amount of solar radiation added to the panel when $+60^{\circ}<\theta_{z}<+90^{\circ}$ equal to the amount of direct solar radiation received by the surface $Q_{1}$. To know what is added to the panel we will calculate the ratio $\mathrm{Q}_{1} / \mathrm{Q}_{2}$.

According to the Fig. 11 we have:

$$
\sin \theta_{z}=\frac{B^{\prime}}{C^{\prime}}
$$

and

$$
\tan \theta_{1}=\frac{C^{\prime}}{A}
$$

According to the Eq. (6), the Eq. (5) becomes: $\sin \theta_{z}=\frac{B^{\prime}}{A \tan \theta_{1}}$ so:

$$
\frac{B^{\prime}}{A}=\sin \theta_{z} \times \tan \theta_{1}
$$

To go to the surface, just multiply by E, so Eq. (7) becomes:

$$
\frac{B^{\prime}}{A}=\frac{B^{\prime} E}{A E}=\frac{Q_{1}}{Q_{2}}=\sin \theta_{z} \times \tan \theta_{1}
$$

According to the Fig. 11 we have: $\theta_{1}=\frac{\pi}{2}-\theta_{z}$ the Eq. (8) become:

$$
\frac{Q_{1}}{Q_{2}}=\sin \theta_{z} \times \cot \theta_{z}
$$

The Table. 1 shows for each degree of $\theta_{\mathrm{z}}$ the total quantity received by the surface of the solar panel compared to $I_{o n}$.

- The first column is the calculation of $I_{o}$ compared to $I_{o n}$ according to Eq. (1).

- The second column is the calculation of the ratio $\frac{\mathrm{Q}_{1}}{\mathrm{Q}_{2}}$ added to the solar panel according to Eq. (9).

- The third column is the total received by the Western Solar plus Mirror model.

Note: We can notice that the quantity added by reflection of solar radiation is the same quantity received directly by the panel; it is due to the
Table 1. The quantity in percentage of a direct solar radiation of the model (PV plus the West mirror)

\begin{tabular}{|r|r|r|r|}
\multicolumn{2}{|c|}{ compared to the $I_{\text {on }}$ with $+60^{\circ}<\theta_{\mathrm{z}}<+90^{\circ}$} \\
$\begin{array}{c}\text { Angle } \\
\text { In } \\
\text { degrees }\end{array}$ & $\begin{array}{c}\mathbf{I}_{\mathbf{o}} \\
\text { (compared } \\
\text { to } \mathbf{I}_{\text {on }} \text { ) }\end{array}$ & $\begin{array}{c}\mathbf{Q}_{\mathbf{1}} / \mathbf{Q}_{\mathbf{2}} \\
\text { (compared } \\
\text { to } \mathbf{I}_{\text {on }} \text { ) }\end{array}$ & $\begin{array}{c}\text { Total } \\
\text { (compared to } \\
\mathbf{I}_{\text {on }} \text { ) }\end{array}$ \\
\hline 90 & $0,00 \%$ & $0,00 \%$ & $0,00 \%$ \\
\hline 85 & $8,72 \%$ & $8,72 \%$ & $17,43 \%$ \\
\hline 80 & $17,36 \%$ & $17,36 \%$ & $34,73 \%$ \\
\hline 75 & $25,88 \%$ & $25,88 \%$ & $51,76 \%$ \\
\hline 70 & $34,20 \%$ & $34,20 \%$ & $68,40 \%$ \\
\hline 65 & $42,26 \%$ & $42,26 \%$ & $84,52 \%$ \\
\hline 60 & $50,00 \%$ & $50,00 \%$ & $100,00 \%$ \\
\hline
\end{tabular}

perpendicular position of mirror in relation to the panel and also the rate of reflection which is equal to 1. the average of the amount of solar radiation received by the system compared to $\mathrm{I}_{\text {on }}$ when $60^{\circ}<\theta_{\mathrm{z}}<+90^{\circ}$ is:

$$
\begin{aligned}
& I_{o\left(60^{\circ}<\theta_{z}<90^{\circ}\right)} \\
& =I_{\text {on }} \\
& \times\left[\frac{\sum_{\theta_{z}=60^{\circ}}^{90^{\circ}}\left(\cos \theta_{z}\right)}{30}\right. \\
& \left.+\quad \frac{\left.\sum_{\theta_{z}=60^{\circ}\left(\frac{Q_{1}}{Q_{2}}\right)}^{30}\right]}{30}\right] \\
& =51.14 \% I_{\text {on }}
\end{aligned}
$$

\subsection{2. when $0^{\circ}<\theta_{z}<+60^{\circ}$}

At $0^{\circ}<\theta_{z}<+60^{\circ}$ the amount of solar radiation reflected by the mirror (represented in Fig. 12 by the surface $Q_{1}=B E$ ) covers an area less than the surface of the panel (shown in the Fig. 12 by the surface $\left.\mathrm{A}^{\prime} \mathrm{E}\right)$. Therefore, we will calculate the ratio $Q_{1} / Q_{2}$, which allows us to know the ratio of the amount of irradiation reflected, compared to the panel surface.

According to the Fig. 12:

$$
\frac{Q_{1}}{Q_{2}}=\frac{B E}{A E}=\frac{B}{A}
$$

According to the Fig. 12 we have:

$$
\sin \theta_{z}=\frac{B}{C}
$$

According to the Eq. (4) and the Eq. (12), the Eq. (11) become: 
Table 2. The quantity in percentage of a direct solar radiation of the model (PV plus the West mirror) compared to the $I_{\text {on }}$ with $0^{\circ}<\theta_{z}<+60^{\circ}$

\begin{tabular}{|c|r|r|r|}
\hline $\begin{array}{c}\text { Angle } \\
\text { In } \\
\text { degrees }\end{array}$ & $\begin{array}{c}\mathbf{I}_{\mathbf{o}} \\
\text { (compared } \\
\text { to } \mathbf{I}_{\mathbf{o n}} \text { ) }\end{array}$ & $\begin{array}{c}\mathbf{Q}_{\mathbf{1}} / \mathbf{Q}_{\mathbf{2}} \\
(\text { compared } \\
\text { to } \mathbf{I}_{\mathbf{o n}} \text { ) }\end{array}$ & $\begin{array}{c}\text { Total } \\
\text { (compared to } \\
\mathbf{I}_{\mathbf{o n}} \text { ) }\end{array}$ \\
\hline 5 & $57,36 \%$ & $47,27 \%$ & $104,62 \%$ \\
\hline 50 & $64,28 \%$ & $44,20 \%$ & $108,48 \%$ \\
\hline 45 & $70,71 \%$ & $40,80 \%$ & $111,51 \%$ \\
\hline 40 & $76,60 \%$ & $37,09 \%$ & $113,69 \%$ \\
\hline 35 & $81,92 \%$ & $33,10 \%$ & $115,01 \%$ \\
\hline 30 & $86,60 \%$ & $28,85 \%$ & $115,45 \%$ \\
\hline 25 & $90,63 \%$ & $24,39 \%$ & $115,02 \%$ \\
\hline 20 & $93,97 \%$ & $19,73 \%$ & $113,70 \%$ \\
\hline 15 & $96,59 \%$ & $14,93 \%$ & $111,53 \%$ \\
\hline 10 & $98,48 \%$ & $10,02 \%$ & $108,50 \%$ \\
\hline 5 & $99,62 \%$ & $5,03 \%$ & $104,65 \%$ \\
\hline 0 & $100,00 \%$ & $0,00 \%$ & $100,00 \%$ \\
\hline
\end{tabular}

$$
\frac{Q_{1}}{Q_{2}}=\frac{C \sin \theta_{z}}{\frac{C}{0.577}}=0.577 \sin \theta_{z}
$$

The Table. 2 shows for each degree of $\theta_{z}$ the total quantity received by the surface of the solar panel compared to $I_{o n}$.

- The first column is the calculation of $I_{o}$ compared to $I_{\text {on }}$ according to Eq. (1)

- The second column is the calculation of the ratio $\frac{\mathrm{Q}_{1}}{\mathrm{Q}_{2}}$ added to the solar panel according to Eq. (13).

- The third column is the total received by the Western Solar plus Mirror model.

According to the Eq. (1) and Eq. (12), the average of the amount of solar radiation received by the system compared to $I_{o n}$ when $0^{\circ}<\theta_{z}<+60^{\circ}$ is:

$$
\begin{aligned}
& I_{o\left(0^{\circ}<\theta_{z}<60^{\circ}\right)} \\
& =I_{o n} \times\left[\frac{\sum_{\theta_{z}=0^{\circ}}^{60^{\circ}}\left(\cos \theta_{z}\right)}{60}+\frac{\sum_{\theta_{z}=0^{\circ}}^{60^{\circ}}\left(Q_{2}\right)}{60}\right] \\
& =\quad 110.25 \% I_{o n}
\end{aligned}
$$

\subsubsection{The daily amount of direct solar irradiation compared to $I_{o n}$}

What happens with the first mirror in the West is happening with the second in the East. When the sun arrives at noon, the first mirror goes down and the second one goes up, the same quantity reflected by the first mirror is reflected by the second since the system is symmetrical. The graph below which is illustrated in Fig. 13, shows the amount of solar irradiation received by the system (panel plus mirrors) compared to the amount received by the

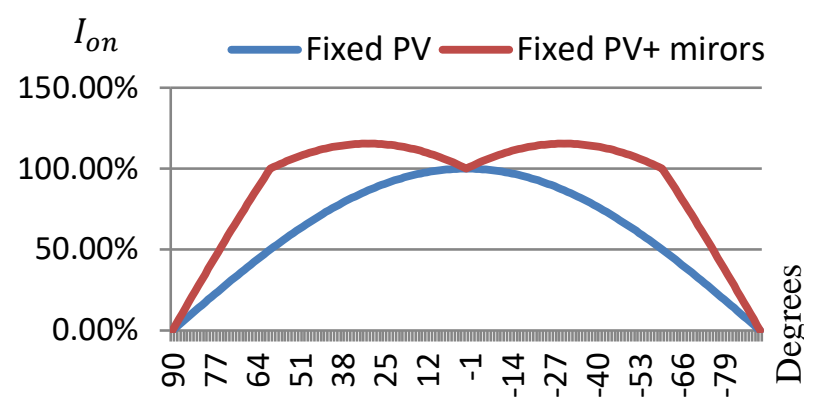

Figure. 13 The amount of direct solar irradiation of fixed PV and PV with mirrors compared to $I_{o n}$

same surface in fixed mode without the proposed model.the equinox day compared to the amount received if the panel surface is normal to solar radiation is:

$$
\begin{aligned}
& I_{o\left(0^{\circ}<\theta_{z}<90^{\circ}\right)} \\
& =I_{o\left(0^{\circ}<\theta_{z}<60^{\circ}\right)} \\
& +I_{o\left(60^{\circ}<\theta_{z}<90^{\circ}\right)} \\
& =I_{\text {on }}\left\{\left[\frac{\sum_{\theta_{z}=0^{\circ}}^{60^{\circ}}\left(\cos \theta_{z}\right)}{60}+\frac{\sum_{\theta_{z}=0^{\circ}}^{60^{\circ}}\left(Q_{2}\right)}{60}\right]\right. \\
& \left.+\left[\frac{\sum_{\theta_{z}=60^{\circ}}^{90^{\circ}}\left(\cos \theta_{z}\right)}{30}+\frac{\sum_{\theta_{z}}^{90^{\circ}}=60^{\circ}\left(\frac{Q_{1}}{Q_{2}}\right)}{30}\right]\right\} \\
& =90.11 \% I_{\text {on }}
\end{aligned}
$$

According to Eq. (2) and Eq. (15), the amount of direct solar irradiation that the system receives on the equinox day compared to the amount received by a fixed PV is $41.9 \%$.

\section{Note}

- Since the system is symmetrical, this percentage calculated in the morning will be the same in the afternoon, so the result of Eq. (15) is the daily ratio calculated for the model together on the day of the equinox.

- The results of the theoretical calculations clearly show that the quantity received by our proposed model is almost similar to that received by a surface normal to solar radiation (a surface with perfect tracking).

- In the theoretical study we considered that the rate of reflections of the mirrors $\mathrm{R}=1$, while actually Mirrors never reflect the same quantity of solar radiation, there is always loss depending on the rate of reflections of the mirrors used.

\subsection{Modification of the proposed model according to the different seasons of the year}




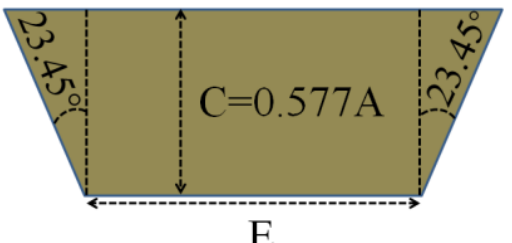

Figure. 14 The trapezoidal shape of mirror

Note that the dimension of the added mirrors is calculated on the day of the equinox when the sun is on the celestial equator, we will modify both of its mirrors to reflect the same amount of solar irradiation reflected during the equinox. At the winter solstice, the sun is over the Tropic of Capricorn, the lowest path in the sky as shown in Fig. 3. The angle between the Tropic of Capricorn and the celestial equator is $23.45^{\circ}$. Likewise, when the sun is over the Tropic of Cancer, the highest path in the sky as shown in Fig. 3 , the angle between the Tropic of Cancer and the celestial equator is $23.45^{\circ}$. That is, at the winter or summer solstice, part of the solar radiation is reflected outside the panel. In order to correct this, it is sufficient that each mirror is of a trapezoidal shape as shown in Fig. 14.

\section{The simulation}

In order to validate the results of the proposed model, we will run a simulation using the PVSyst software.

The simulation will be in two parts:

- The 1st part is a comparison between the hourly gain as a percentage of the quantity of direct irradiation received by the proposed model and that received by a two-axis solar tracker on the day of the equinox, in order to validate the results of the Theoretical study.

- While the 2nd part will be a calculation by simulation of the amount of annual direct irradiation of the proposed model and compare it with that received by a two-axis tracking system and that received with a panel in fixed mode.

The model selected for the simulation: A single panel of 50Wp 15v Si-mono Mono 50Wp 36 ones. In order to focus on direct solar radiation, we set the common value of Albedo to 0 for all objects in the simulation (cancellation of ground reflection or objects during simulation).

\subsection{Hourly comparison of direct solar irradiation during the equinox day}

Since the theoretical calculations are made during the equinox day, we will choose for the simulation March 21, the spring equinox day. In order to

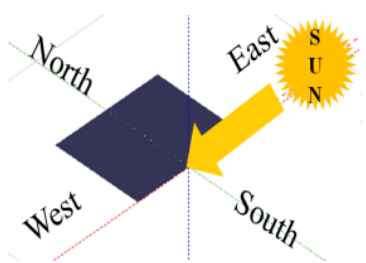

(a)

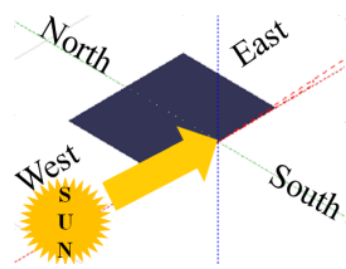

(b)

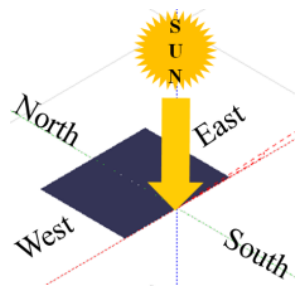

(c)

Figure. 15 optimal installation of a PV in fixed mode at a location on the equator on the day of the equinox: (a)

The sun rises from the geographic east, (b) The sun

rises from the geographic west, and (c) The sun at the zenith

Table 3. The hourly quantity of direct solar irradiation incident on the PV in fixed mode on March 21

\begin{tabular}{|c|c|}
\hline Hour & Beam Eff $\left(\mathbf{W} / \mathbf{m}^{2}\right)$ \\
\hline $06: 00$ & 0,4259 \\
\hline $07: 00$ & 60,613 \\
\hline $08: 00$ & 263,37 \\
\hline $09: 00$ & 474,14 \\
\hline $10: 00$ & 676,98 \\
\hline $11: 00$ & 804,46 \\
\hline $12: 00$ & 584,91 \\
\hline $13: 00$ & 660,35 \\
\hline $14: 00$ & 383,41 \\
\hline $15: 00$ & 349,89 \\
\hline $16: 00$ & 29,811 \\
\hline $17: 00$ & 40,23 \\
\hline
\end{tabular}

simplify this 1 st part of the simulation, we will choose a location on the Earth's equator (Location; Wangata. Country; DR Congo. Latitude; $0.01^{\circ}$. Longitude ; $18.2327^{\circ}$. Altitude ; $318 \mathrm{~m}$ ).

On the earth's equator and during an equinox day, the sun rises exactly from the geographic east as shown in Fig. 15 (a) and sets in the geographic west as shown in Fig. 15 (b) passing through the zenith at noon as shown in Fig. 15 (c). So for a PV installation in fixed mode we have to put the horizontal panel on the ground $\left(\right.$ Tilt $=$ latitude $=0^{\circ}$ ).

The problem with simulation is that we do not have the ability to introduce a mirror to the PV. It is for this reason that we developed a three-step method, in order to have the same results of our mirror model. Let us take into account that the model proposed in this study contains:

- $\quad$ A PV in fixed mode (inclination = latitude of location $=0^{\circ}$ ).

- A model that replaces the mirror of the West. 
- A model that replaces the eastern mirror.

We will start by calculating the amount of direct solar irradiation of a PV in fixed mode as shown in Fig. 16. The Table. 3 shows us the hourly amount of direct solar irradiation incident on the collector (Beam Eff in W/m²) on March 20 (Spring Equinox).

\section{- The model that replaces the West mirror;}

The proposed model contains a mirror west of PV activated in the morning and deactivated in the afternoon, and another mirror east of PV activated in the afternoon and deactivated in the morning as shown in Fig. 9. We are going to replace the first mirror west of the PV with a wall with an opening on top, this opening will have the same surface as that of the 1st mirror as shown in Fig. 16. Normally what is reflected by the 1st mirror will be totally or partially depending on the position of the sun absorbed by the main panel, it is an additional quantity added in the morning by the western mirror, if we put a panel behind the wall opening of the same dimension as the main panel as shown in Fig. 16, this will receive the same amount as that transmitted by the west mirror to the main panel. In this way, we can calculate by simulation the quantity of direct solar irradiation added to the main panel due to the reflection of the 1 st mirror. In order to negate the existence of this pattern in the afternoon, we will completely cover the panel behind the wall as shown in Fig. 16.

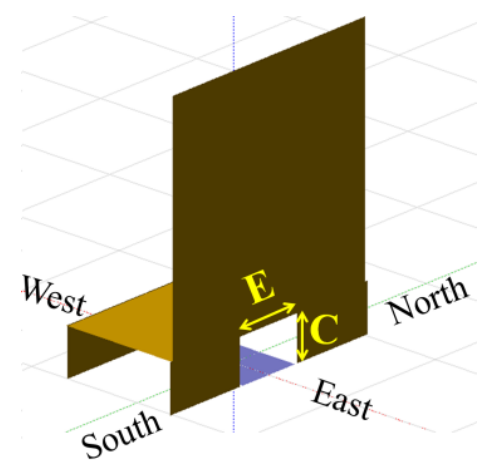

Figure. 16 The model proposed to replace the western mirror

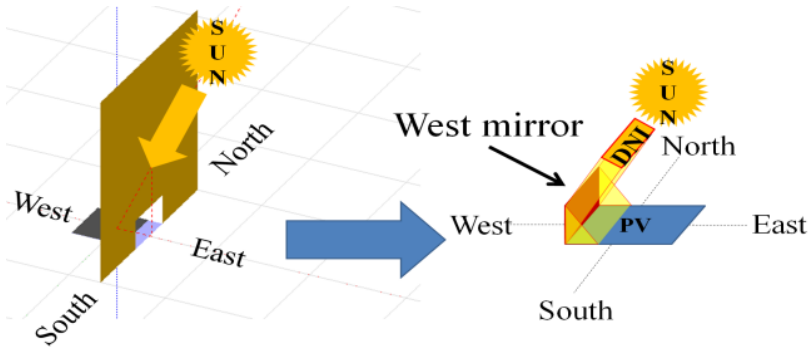

Figure. 17 The model used in the simulation which replaces the Western mirror when $0^{\circ}<\theta_{z}<+60^{\circ}$

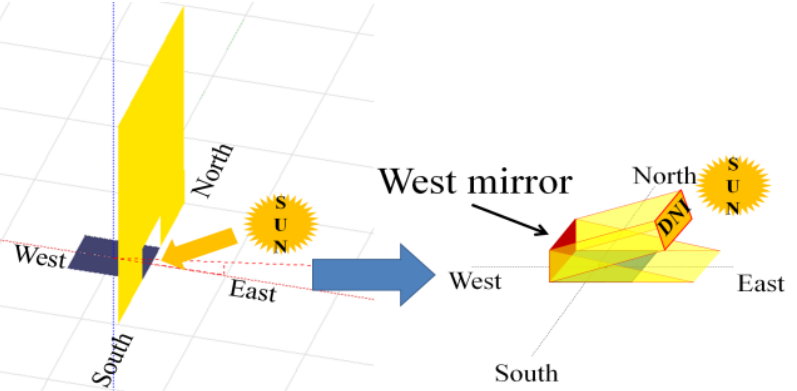

Figure. 18 The model used in the simulation that replaces the eastern mirror when $60^{\circ}<\theta_{z}<+90^{\circ}$

Table 4. The hourly amount of direct solar irradiation incident on the model which replaces the western mirror on March 21

\begin{tabular}{|c|c|}
\hline Hour & Beam Eff $\left(\mathbf{W} / \mathbf{m}^{\mathbf{2}}\right)$ \\
\hline $06: 00$ & 0,4259 \\
\hline $07: 00$ & 60,613 \\
\hline $08: 00$ & 232,82 \\
\hline $09: 00$ & 242,35 \\
\hline $10: 00$ & 194,39 \\
\hline $11: 00$ & 95,381 \\
\hline $12: 00$ & 0 \\
\hline $13: 00$ & 0 \\
\hline $14: 00$ & 0 \\
\hline $15: 00$ & 0 \\
\hline $16: 00$ & 0 \\
\hline $17: 00$ & 0 \\
\hline
\end{tabular}

The Fig. 17 and Fig. 18 clearly show us that this model of an opening wall with a panel behind plays the same role of the western mirror.

At $0^{\circ}<\theta_{z}<+60^{\circ}$ the reflected quantity of direct solar radiation is totally absorbed by a part of the main PV panel and it is the same quantity absorbed by the panel installed behind the opening in case of replacement of the mirror by the wall, as shown in Fig. 17.

At $60^{\circ}<\theta_{z}<+90^{\circ}$ the reflected quantity of direct solar radiation is partially absorbed by the main panel and it is the same quantity absorbed by the panel installed behind the opening if the mirror is replaced by the Wall, as shown in Fig. 18.

The Table. 4 shows the amount of the direct irradiation schedule incident on the PV located behind the wall opening as shown in Fig. 16.

\section{- The model that replaces the East mirror;}

the same principle for the East mirror devoted to the afternoon. It will be replaced in the simulation by the model shown in Fig. 19.

The Table. 5 shows the hourly amount of direct solar irradiation incident on the sensor located behind the wall with opening as shown in Fig. 19. 


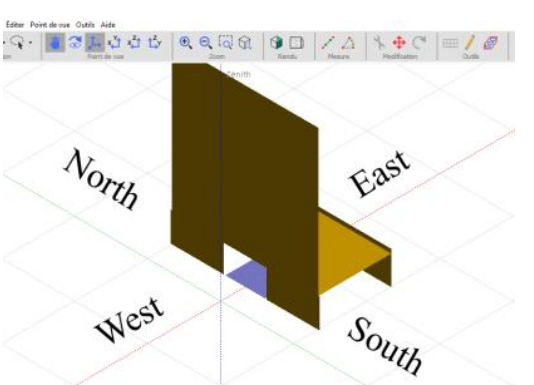

Figure. 19 The model used in the simulation that replaces the east Mirror

Table 5. The hourly amount of direct solar irradiation incident on the model which replaces the eastern mirror on March 21

\begin{tabular}{|c|c|}
\hline Hour & Beam Eff $\left(\mathbf{W} / \mathbf{m}^{2}\right)$ \\
\hline $06: 00$ & 0 \\
\hline $07: 00$ & 0 \\
\hline $08: 00$ & 0 \\
\hline $09: 00$ & 0 \\
\hline $10: 00$ & 0 \\
\hline $11: 00$ & 16,419 \\
\hline $12: 00$ & 120,14 \\
\hline $13: 00$ & 140,6 \\
\hline $14: 00$ & 219,58 \\
\hline $15: 00$ & 29,257 \\
\hline $16: 00$ & 40,23 \\
\hline $17: 00$ & \\
\hline
\end{tabular}

Table 6. The hourly amount of direct solar irradiation incident on the proposed model on March 21

\begin{tabular}{|c|c|}
\hline Hour & Beam Eff $\left(\mathbf{W} / \mathbf{m}^{2}\right)$ \\
\hline $06: 00$ & 0,8518 \\
\hline $07: 00$ & 121,226 \\
\hline $08: 00$ & 496,19 \\
\hline $09: 00$ & 716,49 \\
\hline $10: 00$ & 871,37 \\
\hline $11: 00$ & 899,841 \\
\hline $12: 00$ & 601,329 \\
\hline $13: 00$ & 780,49 \\
\hline $14: 00$ & 524,01 \\
\hline $15: 00$ & 569,47 \\
\hline $16: 00$ & 59,068 \\
\hline $17: 00$ & 80,46 \\
\hline
\end{tabular}

Finally, to simulate the hourly quantity of direct solar irradiation of our model proposed on March 21, it suffices to add the results of Table. 4 and 5 to the results of Table. 3 (The quantity received by the main PV ).

These results which are illustrated in the Table. 6 will be compared with those simulated from a PV having the same characteristics (A single panel of 50Wp 15v Si-mono Mono 50Wp 36 ones) mounted on a two-axis tracker as shown in Table. 7. On March 21 , the sun rises at 6:00 a.m. in the morning and sets at 6:00 p.m. in the evening. So every hour, the sun
Table 7. The comparison between the results of the theoretical and simulation gain on March 21

\begin{tabular}{|c|c|c|c|c|c|}
\hline Hour & Angle & $\begin{array}{c}\text { PVwith } \\
\text { Our } \\
\text { model } \\
\text { (Beam } \\
\text { Eff) }\end{array}$ & $\begin{array}{c}\text { PV with } \\
\text { tracking } \\
\text { mode } \\
\text { (Beam } \\
\text { Eff) }\end{array}$ & $\begin{array}{c}\text { The } \\
\text { simulation } \\
\text { gain in } \\
\text { percentage }\end{array}$ & $\begin{array}{c}\text { Theoretical } \\
\text { percentage } \\
\text { gain }\end{array}$ \\
\hline $\mathbf{6 : 0 0}$ & $\mathbf{9 0}^{\circ}$ & 0,8 & 10,7 & $7,92 \%$ & $0 \%$ \\
\hline $\mathbf{7 : 0 0}$ & $\mathbf{7 5}^{\circ}$ & 121,2 & 238,1 & $50,90 \%$ & $51,76 \%$ \\
\hline $\mathbf{8}: 00$ & $\mathbf{6 0}^{\circ}$ & 496,1 & 505,1 & $98,23 \%$ & $100 \%$ \\
\hline $\mathbf{9 : 0 0}$ & $\mathbf{4 5}^{\circ}$ & 716,4 & 647,4 & $110,66 \%$ & $111,51 \%$ \\
\hline $\mathbf{1 0}: \mathbf{0 0}$ & $\mathbf{3 0}^{\circ}$ & 871,3 & 761,9 & $114,36 \%$ & $115,45 \%$ \\
\hline $\mathbf{1 1 : 0 0}$ & $\mathbf{1 5}^{\circ}$ & 899,8 & 823,4 & $109,28 \%$ & $111,53 \%$ \\
\hline $\mathbf{1 2}: \mathbf{0 0}$ & $\mathbf{0}^{\circ}$ & 601,3 & 580,9 & $103,51 \%$ & $100 \%$ \\
\hline $\mathbf{1 3 : 0 0}$ & $\mathbf{- 1 5}^{\circ}$ & 780,4 & 694,4 & $112,40 \%$ & $111,53 \%$ \\
\hline $\mathbf{1 4 : 0 0}$ & $\mathbf{- 3 0}^{\circ}$ & 524,0 & 458,2 & $114,35 \%$ & $115,45 \%$ \\
\hline $\mathbf{1 5 : 0 0}$ & $\mathbf{- 4 5}^{\circ}$ & 569,4 & 530,4 & $107,35 \%$ & $111,51 \%$ \\
\hline $\mathbf{1 6 : 0 0}$ & $\mathbf{- 6 0}^{\circ}$ & 59,0 & 70,1 & $84,24 \%$ & $100 \%$ \\
\hline $\mathbf{1 7}: \mathbf{0 0}$ & $\mathbf{- 7 5}^{\circ}$ & 80,4 & 270,7 & $29,71 \%$ & $51,76 \%$ \\
\hline
\end{tabular}

moves $15^{\circ}$ in the sky (apparent movement of the sun). Which is illustrated in the Table. 7, we will compare the results of the percentage gain calculated by simulation, with those calculated theoretically in Table. 1 and Table. 2.

Table. 7 clearly shows that the simulation results for the equinox day of March 21 are almost the same as those calculated theoretically.

\subsection{The annual gain in direct solar irradiation offered by the proposed model}

We will continue the simulation to cover the whole year and compare the results obtained with a fixed system and that with a solar tracker. Since we will end the work with a real experience, we choose the city of Khemisset in Morocco as the simulation site (a place far from the equator). Fig. 20 shows the model used in the simulation, it is a fixed installation inclined at $34^{\circ}$ (Khemisset's latitude) with an opening wall of the same size and the same shape as the mirror calculated in the previous chapter, while taking into account the modification of the mirror

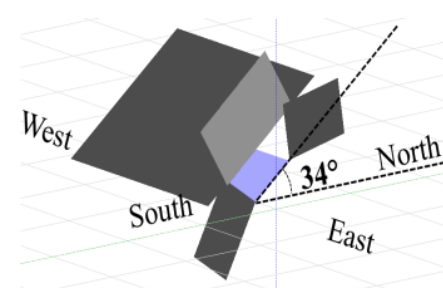

(a)

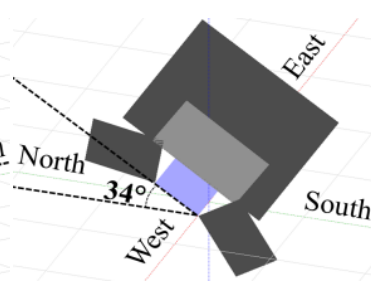

(b)
Figure. 20 The model used in the simulation of a khemisset city (latitude $=34^{\circ}$ ): (a) the model used to replace the Western mirror and (b) the model used to replace the eastern mirror 
Table 8 . The annual quantity of direct solar radiation incident on the proposed model in the Khemisset city

\begin{tabular}{|c|c|c|c|c|}
\hline Month & $\begin{array}{l}\text { Fixed PV } \\
\text { BeamEff }\end{array}$ & $\begin{array}{c}\text { Module } \\
\text { that } \\
\text { replaces the } \\
\text { Western } \\
\text { mirror } \\
\text { BeamEff } \\
\end{array}$ & 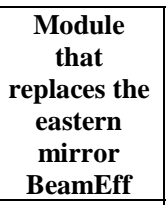 & $\begin{array}{l}\text { PV module } \\
\text { BeamEff }\end{array}$ \\
\hline 1 & 146,62 & 25,65 & 22,741 & 195,011 \\
\hline 2 & 135,45 & 24,885 & 23,793 & 184,128 \\
\hline 3 & 90,172 & 16,921 & 16,337 & 123,43 \\
\hline 4 & 114,1 & 21,497 & 21,871 & 157,468 \\
\hline 5 & 137,68 & 23,469 & 25,195 & 186,344 \\
\hline 6 & 147,67 & 25,518 & 26,52 & 199,708 \\
\hline 7 & 150,03 & 24,681 & 27,699 & 202,41 \\
\hline 8 & 159,85 & 28,918 & 29,852 & 218,62 \\
\hline 9 & 121,76 & 20,881 & 23,61 & 166,251 \\
\hline 10 & 132,38 & 24,006 & 21,266 & 177,652 \\
\hline 11 & 122,46 & 20,5 & 19,103 & 162,063 \\
\hline 12 & 125,19 & 19,914 & 19,485 & 164,589 \\
\hline Total & 1583,4 & 276,84 & 277,47 & 2137,71 \\
\hline
\end{tabular}

Table 8 . The annual quantity of direct solar radiation incident on the proposed model in the Khemisset city

\begin{tabular}{|c|c|c|c|c|}
\hline $\begin{array}{c}\text { Mont } \\
\text { h }\end{array}$ & $\begin{array}{c}\text { Fixed } \\
\text { PV } \\
\text { BeamEf } \\
\text { f }\end{array}$ & $\begin{array}{l}\text { Module } \\
\text { that } \\
\text { replaces } \\
\text { the } \\
\text { Western } \\
\text { mirror } \\
\text { BeamEff }\end{array}$ & $\begin{array}{l}\text { Module } \\
\text { that } \\
\text { replaces } \\
\text { the } \\
\text { eastern } \\
\text { mirror } \\
\text { BeamEff }\end{array}$ & $\begin{array}{c}\text { PV } \\
\text { module } \\
\text { BeamEff }\end{array}$ \\
\hline 1 & 146,62 & 25,65 & 22,741 & 195,011 \\
\hline 2 & 135,45 & 24,885 & 23,793 & 184,128 \\
\hline 3 & 90,172 & 16,921 & 16,337 & 123,43 \\
\hline 4 & 114,1 & 21,497 & 21,871 & 157,468 \\
\hline 5 & 137,68 & 23,469 & 25,195 & 186,344 \\
\hline 6 & 147,67 & 25,518 & 26,52 & 199,708 \\
\hline 7 & 150,03 & 24,681 & 27,699 & 202,41 \\
\hline 8 & 159,85 & 28,918 & 29,852 & 218,62 \\
\hline 9 & 121,76 & 20,881 & 23,61 & 166,251 \\
\hline 10 & 132,38 & 24,006 & 21,266 & 177,652 \\
\hline 11 & 122,46 & 20,5 & 19,103 & 162,063 \\
\hline 12 & 125,19 & 19,914 & 19,485 & 164,589 \\
\hline Total & 1583,4 & 276,84 & 277,47 & 2137,71 \\
\hline
\end{tabular}

made in order to adapt the model with the sun in summer and winter solstice as shown in Fig. 14 (trapezoidal mirror).

The Table. 8 shows us the results of the block module simulation. In order to validate these results by comparison, we are going to simulate the same PV parameters in fixed mode and in tracking mode. This comparison is shown in Table. 9.

The results clearly show us that the proposed model offers an efficiency of about $94 \%$ compared to the solar tracker system and 35\% compared to the fixed mode PV, This gain is almost similar to document production gain [9] and close to what was
Table 9. The comparison of the annual yield between the proposed model, the fixed mode and the followed

\begin{tabular}{|c|c|c|c|}
\hline Month & $\begin{array}{c}\text { Fixed PV } \\
\text { BeamEff }\end{array}$ & $\begin{array}{c}\text { PV with } \\
\text { tracking } \\
\text { mode } \\
\text { BeamEff }\end{array}$ & $\begin{array}{c}\text { PV with the } \\
\text { proposed } \\
\text { model } \\
\text { BeamEff }\end{array}$ \\
\hline 1 & 146,62 & 199,07 & 195,011 \\
\hline 2 & 135,45 & 181,35 & 184,128 \\
\hline 3 & 90,172 & 122,1 & 123,43 \\
\hline 4 & 114,1 & 169,25 & 157,468 \\
\hline 5 & 137,68 & 212,74 & 186,344 \\
\hline 6 & 147,67 & 247,72 & 199,708 \\
\hline 7 & 150,03 & 237,1 & 202,41 \\
\hline 8 & 159,85 & 236,36 & 218,62 \\
\hline 9 & 121,76 & 168,67 & 166,251 \\
\hline 10 & 132,38 & 174,39 & 177,652 \\
\hline 11 & 122,46 & 160,3 & 162,063 \\
\hline 12 & 125,19 & 168,78 & 164,589 \\
\hline total & $\mathbf{1 5 8 3 , 4}$ & $\mathbf{2 2 7 7 , 8}$ & $\mathbf{2 1 3 7 , 7 1}$ \\
\hline
\end{tabular}

received by the solar follower model proposed by the documents $[10,11]$. This allows us to conclude that this system gives almost the same performance as that of a two-axis solar tracker. We can also conclude that the results of the simulation are very close to the results obtained by the theory.

\section{Making the prototype}

The system in principle has only one movement, this is the moment when the two mirrors are reversed at noon or in the evening. We can use two motors, in this way each mirror will have an independent movement of the other, which will be useful if we want to lower the two mirrors to the faith in the case where the wind blows stronger in order to protect the structure. We can also use a single motor for this manipulation, the moment the motor removes the $1 \mathrm{st}$ mirror downwards, the 2nd goes up and vice versa. Alternatively, we can also use a single mirror with two reflective surfaces, in the morning the mirror is

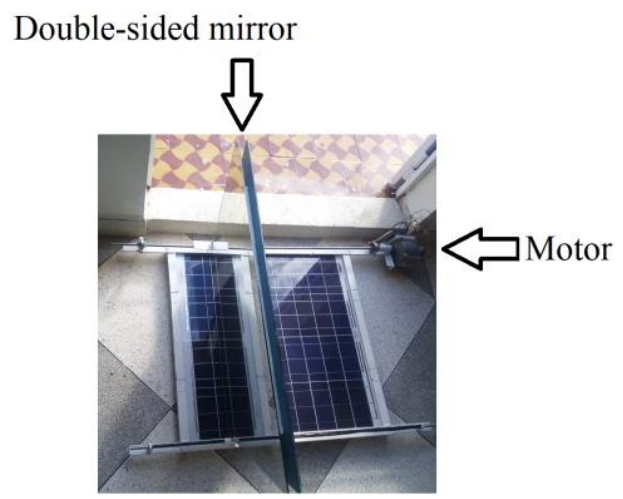

Figure. 21 Double-sided mirror with the proposed prototype 


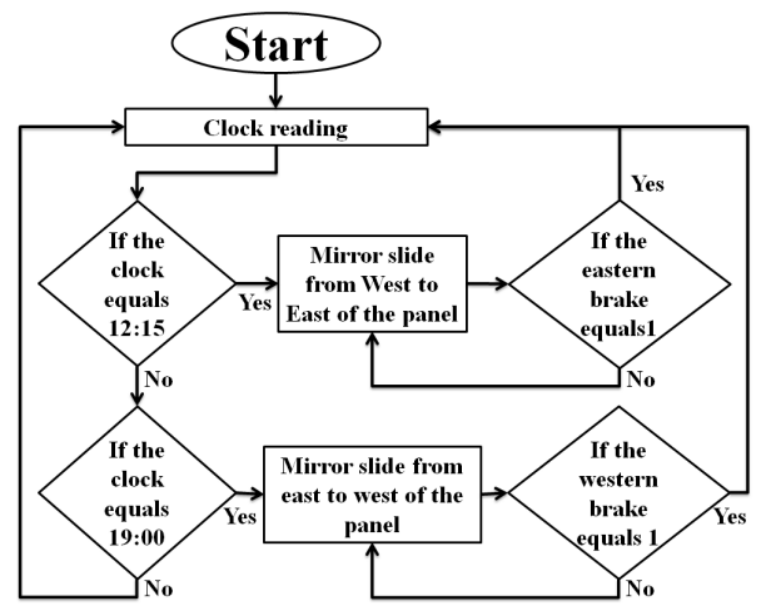

Figure. 22 The organization chart that controls the prototype

Table 10. The cost of the prototype

\begin{tabular}{|l|c|}
\hline \multicolumn{1}{|c|}{ Equipment } & Price $\mathbf{( \$ )}$ \\
\hline Arduino Uno & 3,5 \\
\hline ULN 2804 & 1,7 \\
\hline Clock DS3231 & 1,2 \\
\hline $\begin{array}{l}\text { Unipolar stepper motor } \\
\text { with a pigeon reduction }\end{array}$ & 8 \\
\hline The structure & 6,5 \\
\hline Mirrors & 6 \\
\hline TOTAL & 26,9 \\
\hline
\end{tabular}

to the West of the panel, while in the afternoon the mirror slides via a motor with a linear movement towards the East of the panel as shown in the Fig. 21, this is the principle chosen for our prototype.

The motor chosen is of the unipolar stepper (type 55SI-25DAYA) with a pigeon reduction gear and a control system with an Arduino uno. The motor is controlled by ULN2804 circuit. Since the prototype will have a single motor, we have two sliding movements, one at noon when the mirror passes from the East to the West of the panel (we take 12h15min GMT as the average time of noon), the other on evening in the opposite direction (we take 19h00min GMT as average evening time). In order for the system to know the mirror slip moment, we will introduce a clock of type DS3231.

According to Fig. 22, at the start the system reads the contents of the clock and compares it with the $12 \mathrm{~h} 15 \mathrm{~min}$ noon hour, if they are equal, the motor will operate in a way to slide the mirror towards the East until the mirror touches a brake installed at the limit of the eastern path of the panel as shown in the Fig. 21 . The same procedure for evening time $(19 \mathrm{~h} 00 \mathrm{~min})$, the mirror slides until it touches a brake installed at the limit of the westward path of the panel as shown in the Fig. 21.The Table. 10 shows the cost of the prototype realised.
It can be noted that the production cost of our system is low compared to a tracking system either bi-axial or mono-axial. As an example, the prototype proposes by publication [3] with a production cost of $\$ 240$, and the prototype in the publications [6] and [11] with a production cost of about $\$ 100$.

\section{Experience and results}

\subsection{Production}

After the production of the prototype, we carried out an experiment in order to really enhance the proposed model. In the simulation, we compared the amount of radiation absorbed by three installation modes; the fixed mode, the bi-axial tracking mode and finally the model we proposed. The simulation comparison between the proposed and the bi-axial follow-up mode was necessary in order to validate the theoretical results (in theory we calculated the radiation gain of the proposed model compared to a normal surface to solar radiation). The simulation comparison between the proposed model and the one in fixed mode will be necessary for the experimental valorization of the concept, an installation of a photovoltaic panel in fixed mode is a reference of experimental valorization for each proposed model, and we will compare the energy gain produced by our model. The experiment took place in the city of khemisset $\left(\right.$ Latitude $=34^{\circ}$ ) on December 31, 2020, we used two photovoltaic panels with the same

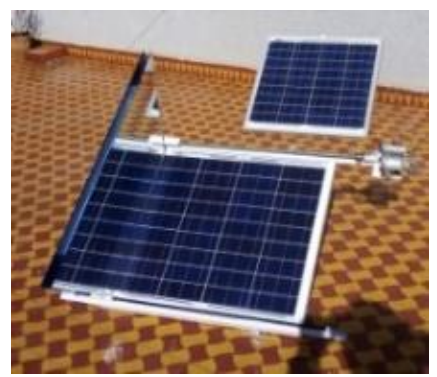

Figure. 23 The model proposed in the day of the experiment

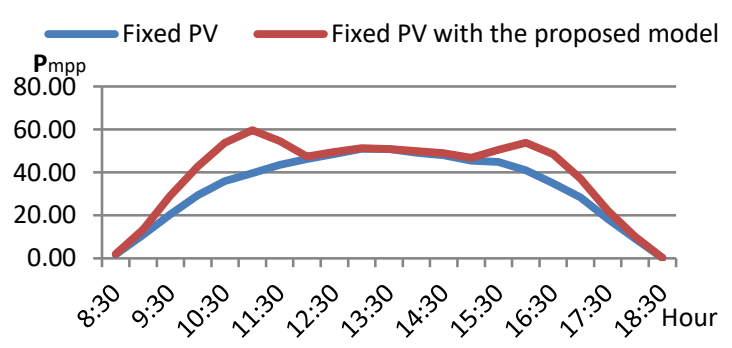

Figure. 24 The comparison of the produced energy between the PV in fixed mode with and without the proposed prototype 
Table 11. The results of the real experience

\begin{tabular}{|r|r|r|r|r|r|r|}
\hline & \multicolumn{4}{|c|}{ Fixed PV } & \multicolumn{2}{|c|}{$\begin{array}{c}\text { Fixed PV with the } \\
\text { proposed model }\end{array}$} \\
\hline Hour & \multicolumn{1}{|c|}{ Voc } & \multicolumn{1}{|c|}{ Iac } & \multicolumn{1}{c|}{ Pmpp } & \multicolumn{1}{|c|}{ Voc } & \multicolumn{1}{l|}{ Iac } & Pmpp \\
\hline $08: 30$ & 18,7 & 0,13 & 1,85 & 19 & 0,14 & 2,02 \\
\hline $09: 00$ & 21 & 0,68 & 10,85 & 21,3 & 0,83 & 13,44 \\
\hline $09: 30$ & 21,4 & 1,26 & 20,49 & 21,9 & 1,75 & 29,13 \\
\hline $10: 00$ & 21,2 & 1,82 & 29,32 & 21,6 & 2,6 & 42,68 \\
\hline $10: 30$ & 21,4 & 2,21 & 35,94 & 21,6 & 3,28 & 53,84 \\
\hline $11: 00$ & 20,9 & 2,5 & 39,71 & 21,1 & 3,72 & 59,65 \\
\hline $11: 30$ & 20,4 & 2,81 & 43,57 & 20,6 & 3,49 & 54,64 \\
\hline $12: 00$ & 20,7 & 2,94 & 46,25 & 20,8 & 3 & 47,42 \\
\hline $12: 30$ & 20,6 & 3,1 & 48,53 & 20,7 & 3,15 & 49,56 \\
\hline $13: 00$ & 20,9 & 3,21 & 50,99 & 20,9 & 3,23 & 51,31 \\
\hline $13: 30$ & 20,7 & 3,23 & 50,81 & 20,7 & 3,23 & 50,81 \\
\hline $14: 00$ & 20,8 & 3,11 & 49,16 & 20,8 & 3,16 & 49,95 \\
\hline $14: 30$ & 20,8 & 3,04 & 48,06 & 20,8 & 3,1 & 49,00 \\
\hline $15: 00$ & 21 & 2,85 & 45,49 & 21,1 & 2,92 & 46,83 \\
\hline $15: 30$ & 20,9 & 2,82 & 44,79 & 21 & 3,16 & 50,43 \\
\hline $16: 00$ & 21,2 & 2,54 & 40,92 & 21,4 & 3,31 & 53,83 \\
\hline $16: 30$ & 21,1 & 2,18 & 34,96 & 21,3 & 3 & 48,56 \\
\hline $17: 00$ & 20,8 & 1,79 & 28,30 & 21,1 & 2,32 & 37,20 \\
\hline $17: 30$ & 20,5 & 1,17 & 18,23 & 21 & 1,39 & 22,18 \\
\hline $18: 00$ & 20,5 & 0,59 & 9,19 & 20,1 & 0,66 & 10,08 \\
\hline $18: 30$ & 15,2 & 0,02 & 0,23 & 15,2 & 0,02 & 0,23 \\
\hline & Total & & $\mathbf{6 9 7 , 6 5}$ & Total & $\mathbf{8 2 2 , 8 2}$ \\
\hline
\end{tabular}

characteristics with a fixed installation (orientation to the south with an inclination equal to the latitude of the place), one with the proposed model, the other not, as shown in Fig. 23. At noon, we reversed the two panels to get accurate results.

The characteristics of the solar panels are:

$\mathrm{V}_{\mathrm{oc}}=22.1 \mathrm{~V}, \mathrm{~V}_{\mathrm{mpp}}=18.2 \mathrm{~V}, \mathrm{Iac}_{\mathrm{c}}=2.95 \mathrm{~A}, \mathrm{Impp}=2.75$ A.

So the form factor is:

$$
\begin{aligned}
F F=\left(V_{m p p} \times I_{m p p}\right) \times\left(V_{o c} \times I_{a c}\right) & \\
& =P_{m p p}\left(V_{o c} \times I_{a c}\right)=0.76
\end{aligned}
$$

So:

$$
P_{m p p}=0.76 \times V_{o c} \times I_{a c}
$$

We measured every $30 \mathrm{~min}$ the Vco and Icc to calculate the power of each photovoltaic panel with Eq. (16). The obtained results are shown in Table. 11 and the graph in Fig. 24.

\subsection{Consumption}

We measured the voltage and the current when the stepper motor is in stop mode, in this case the system power consumption equal:

$$
\begin{aligned}
P_{1}=V_{1}+I_{1}= & 12 v \times 0.012 \mathrm{~A} \\
= & 0.144 \mathrm{~W}
\end{aligned}
$$

And we measured the voltage and the current when the stepper motor is in on mode:

$$
P_{2}=V_{2}+I_{2}=12 v \times 0.091 \mathrm{~A}=1.92 \mathrm{~W}
$$

The double mirrors do the route twice a day, at noon and in the evening. Each route lasts $\mathrm{T}=7 \mathrm{~min}$. According to Eq. (17) and Eq. (18) the total system consumption in 24 hours equal:

$$
\begin{aligned}
P_{\text {Day }}=\left(\frac{P_{1} \times(24 h-2 T)}{24 h}\right) \\
+\left(\frac{P_{2} \times 2 T}{24 h}\right)=0.161 \mathrm{~W}
\end{aligned}
$$

So the prototype consumption is very negligible.

\section{Discussion}

December 31 st, is the day that we chose to perform the experiment, the sun on that day was almost over the tropic of cancer (10 days after the winter solstice), it rose at 8:30 am on the morning and sat at 18:30 (legal time in Morocco: GMT + 1). According to the simulation, the quantity of direct solar irradiation received by our model proposed on December $31 \mathrm{st}$ is $4.45 \mathrm{kWh} / \mathrm{m}^{2}$ / day while with a panel in fixed mode this quantity is $3.44 \mathrm{kWh} / \mathrm{m}^{2} /$ day it means an increase of $29.36 \%$. According to Table. 11, the results of the experiment show an increase in production energy of about $18 \%$ (the measured energy production of the proposed prototype compared to fixed mode PV). In the simulation we considered that the reflection is absolute but in reality, a mirror will never be able to reflect $100 \%$ of incident radiation, especially since the mirrors used in the prototype are very ordinary at a lower cost with a reflection rate of approximately $70 \%(\mathrm{R}=0.7)$, it is for this reason that the yield drops in the case of the experiment, we can also add that the solar panel has a protective glass pane which also consists of a radiation obstacle solar. A general overview on Fig. 24 (daily result of the experiment) and figure 13 (daily result of the theory) clearly shows a Concordance, which leads us to conclude that the actual results are compatible with the theoretical calculations made. This increase in yield measured on the day of the experiment $(18 \%)$ is more than that declared by document [11] that they measured a yield of between 10 and $13.23 \%$ in winter, and also to that measured also by the document [22]. The experiment also confirmed to us the negligible consumption of the system compared to the amount of energy production of the prototype. To this end, 
our proposed model is advantageous by the simplicity of its mechanism, its minimal cost, its negligible energy consumption and a fairly high production.

\section{Conclusion}

Through this document, we have proposed a new simple model introduced to a photovoltaic panel in fixed mode, which allows the latter to increase its efficiency even if the sun is far from the meridian. We started with a theoretical study has shown that the new model will receive almost $41.9 \%$ more of the amount of direct solar irradiation compared to a fixed PV and about $90.11 \%$ compared to a solar tracker panel. Then we simulated the annual yield of the entire model via the PVsys software, which allowed us to obtain almost the same results as the theoretical study. Thereafter, a prototype was produced in order to practically enhance the proposed model. Finally the document is closed by a real experience during a whole day, which revealed an efficiency of more than about $18 \%$ compared to a panel in fixed mode, which allows us to conclude that this simple model is less expensive with an efficiency of energy close to that offered by a solar tracker with all the complexity of its mechanism.

\section{Perspective}

Following the experience already carried out in a single day and in order to validate and generalize the results of the new concept, we will work on the possibility of carrying out the same experiments spread over a period of 6 months, between the spring equinox and the autumn equinox.

\section{Conflicts of Interest}

The authors declare no conflict of interest.

\section{Author Contributions}

Hicham Bouzakri, as the corresponding author, has designed the proposed model of the photovoltaic panel. Also, he has modeled and simulates the proposed system using PVsyst environment. Ahmad Abbou has supervised the written paper and providing the necessary data. Khalid Chennoufi has contributed to the writing and the paper organization. All authors approved the final version.

\section{References}

[1] S. R. Wenham, Applied Photovoltaics, Routledge, 2011

[2] R. El Idrissi, A. Abbou, and M. Mokhlis, "Backstepping Integral Sliding Mode Control
Method for Maximum Power Point Tracking for Optimization of PV System Operation Based on High-Gain Observer", International Journal of Intelligent Engineering and Systems, Vol. 13, No.5, pp. 133-144, 2020.

[3] S. Gutérrez, "Prototype for an Off-grid Photovoltaic System With Low Cost Solar Tracking”, In: Proc. of 2017 IEEE Mexican Humanitarian Technology Conference, Puebla, Mexico, pp. 7-11, 2017.

[4] C. T. Y. Xi, M. Z. Daud, W. M. W. Muda, and M. A. Jusoh, "Development of MicrocontrollerBased Solar Tracking System Using LDR Sensor", Journal of Telecommunication, Electronic and Computer Engineering, Vol. 9, No. 2-7, pp. 71-75, 2017.

[5] H. Wang, T. Luo, Y. Fan, Z. Lu, H. Song, and J. B. Christen, "A Self-powered Single-axis Maximum Power Direction Tracking System With an On-chip Sensor", Solar Energy, Vol. 112, pp. 100-107, 2015.

[6] C. Jamroen, P. Komkum, S. Kohsri, W. Himananto, S. Panupintu, and S. Unkat, "A Low-cost Dual-axis Solar Tracking System Based on Digital Logic Design: Design and Implementation", Sustainable Energy Technologies and Assessments, Vol. 37, pp. 100618, 2020.

[7] Y. Yao, Y. Hu, S. Gao, G. Yang, and J. Du, "A Multipurpose Dual-axis Solar Tracker with Two Tracking Strategies", Renewable Energy, Vol. 72, pp. 88-98, 2014.

[8] M. R. I. Sarker, M. R. Pervez, and R. A. Beg, "Design, Fabrication and Experimental Study of a Novel Two-axis Sun Tracker", International Journal of Mechanical \& Mechatronics Engineering, Vol. 10, No. 01, pp. 13-18, 2010.

[9] S. Abdallah, "The Effect of Using Sun Tracking Systems on the Voltage-current Characteristics and Power Generation of Flat Plate Photovoltaics", Energy Conversion and Management, Vol. 45, No. 11-12, pp. 16711679, 2004.

[10] H. Bouzakri and A. Abbou, "Study and Realization of A Monoaxial Solar Tracker Over an Equatorial Mount", In: Proc. of 7th International Renewable and Sustainable Energy Conference, Agadir, Morocco, pp. 1-6, 2019.

[11] H. Bouzakri and A. Abbou, "Mono-axial Solar Tracker with Equatorial Mount, for an Improved Model of a Photovoltaic Panel", International Journal of Renewable Energy Research, Vol. 10, No. 2, pp. 578-590, 2020. 
[12] H. Tanaka, "Theoretical Analysis of Solar Thermal Collector with a Flat Plate Bottom Booster Reflector", Energy Science and Technology, Vol. 2, No. 2, pp. 26-34, 2011.

[13] P. Malik and S. S. Chandel, "Performance enhancement of multi-crystalline silicon photovoltaic modules using mirror reflectors under Western Himalayan climatic conditions", Renewable Energy, Vol. 154, pp. 966-975, 2020.

[14] V. N. Palaskar, and S. P. Deshmukh, "Design and performance analysis of reflectors attached to commercial PV module", International Journal of Renewable Energy Research, Vol. 4, No. 1, pp. 240-245, 2014.

[15] M. S. Gopinnath, R. Balaji, and V. Kirubakaran, "Cost Effective Methods to Improve the Power Output of a Solar Panel: An Experimental Investigation", In: Proc. of Power and Energy Systems: Towards Sustainable Energy, Bangalore, India, pp. 1-4, 2014.

[16] M. Mansoor, S. P. Simon, K. A. Kumar, K. Sundareswaran, P. S. R. Nayak, and N. P. Padhy, "Impact and economic assessment on solar PV mirroring system-A feasibility report", Energy Conversion and Management, Vol. 203, pp. $112222,2020$.

[17] M. Iqbal, An introduction to solar radiation, Elsevier, 2012. 